\title{
Caecal Volvulus: An Uncommon Disease with Common Presentation
}

\author{
Muhammed $\mathrm{AS}^{1}$, Aye $\mathrm{M}^{2 *}$ and Harshad $\mathrm{R}^{3}$ \\ ${ }^{1}$ Department of surgery, Melaka General Hospital, Malaysia \\ ${ }^{2}$ Department of Medicine, Melaka Manipal Medical College, Malaysia \\ ${ }^{3}$ Medical student of Melaka Manipal Medical College, Malaysia \\ *Corresponding author: Mra Aye Department of Medicine, Malaysia
}

\begin{tabular}{|c|c|}
\hline ARTICLE INFO & ABSTRACT \\
\hline Received: 㓞 June 19, 2019 & Caecal volvulus is rare cause of intestinal obstruction. However, it is imperative to \\
\hline Published: 幽 June 28, 2019 & $\begin{array}{l}\text { know the predisposing factors to make an early diagnosis of cecal volvulus and would } \\
\text { be able to decrease morbidity and mortality. Here, we report a case of caecal volvulus in }\end{array}$ \\
\hline
\end{tabular}

R. Caecal Volvulus: An Uncommon

Disease with Common Presentation.

Biomed J Sci \& Tech Res 19(2)-2019.

BJSTR. MS.ID.003268.

\section{Introduction}

Ceacal volvulus was first noted by Hildanus in the $16^{\text {th }}$ century and later reviewed by Rokitansky in 1837. It is the second most common site for volvulus of intestine. The incidence of cecal volvulus is reported to range from 2.8 to 7.1 per million people per year [1]. It accounts for 1 to $1.5 \%$ of all the adult intestinal obstructions and 25 to $40 \%$ of all volvulus involving the colon. It is axial twisting that involves the caecum, terminal ileum and ascending colon. It is due to incomplete embryological rotation of the bowel or improper developmental fusion is explanation for development of caecal volvulus. There are two prerequisites for caecal volvulus to occur: a segment of mobile caecum and ascending colon and a point of fixation about which torsion may occur [2]. In addition to the prerequisite of a freely mobile caecum, several additional predisposing factors have been implicated in the genesis of caecal volvulus. These include concomitant acute medical problems, pregnancy, distal colonic obstruction, previous laparotomy, and gynaecological procedures [3] (Figure 1). It is reported to be associated with previous abdominal surgery in up to $68 \%$ of cases [4,3]. Appendicitis as a cause of caecal volvulus was first reported by Cochrane in 1929 followed by a few reports [5,6].

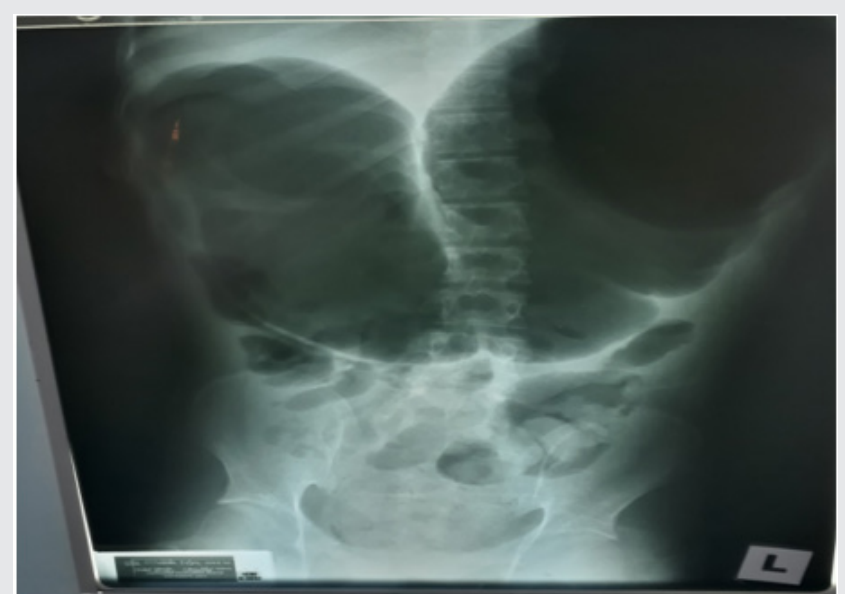

Figure 1: Plain X-ray Abdomen Report: Dilated bowel with coffee bean shape.

Patients with volvulus are commonly elderly, debilitated, and bedridden. Often, the patient has a history of dementia or neuropsychiatric impairment. As a result, only a limited history is available (Figure 2). More than $60-70 \%$ of patients present with 
acute symptoms; the remainder present with subacute or chronic symptoms. A history of chronic constipation is common. The patient may describe previous episodes of abdominal pain, distention, and obstipation, which suggest repeated subclinical episodes of volvulus [7]. The disease predominantly affects the females. If persistent, volvulus will cause vascular compromise which may proceed to caecal gangrene and bowel injury and carries a high mortality, up to $40 \%$. [1]. Conventional radiography demonstrates a disproportionate distention of the right colon against a collapsed left colon, small bowel dilatation and proximal obstruction signs may occur depending on the time of onset [8-10].

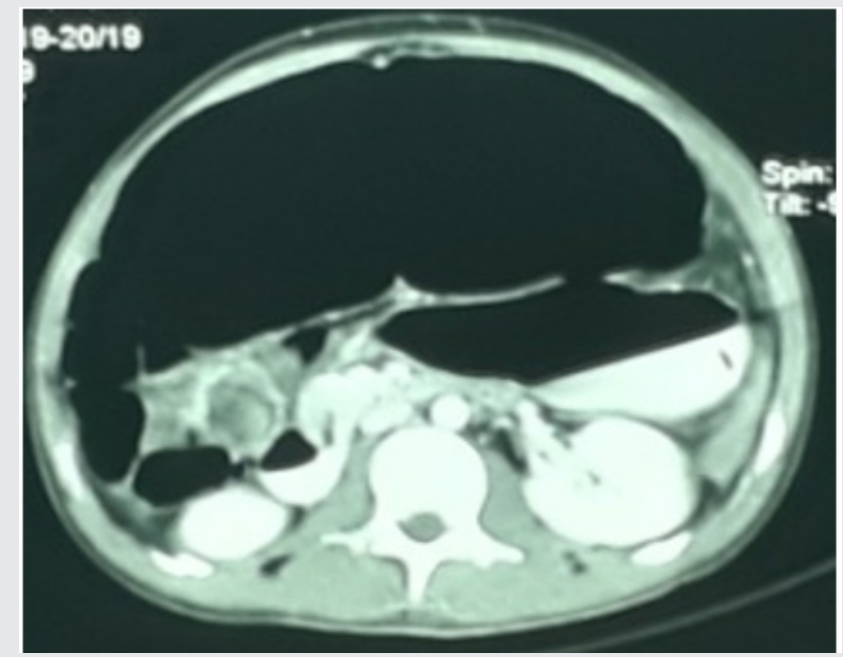

Figure 2: CT Abdomen CT abdomen was reported as follow.

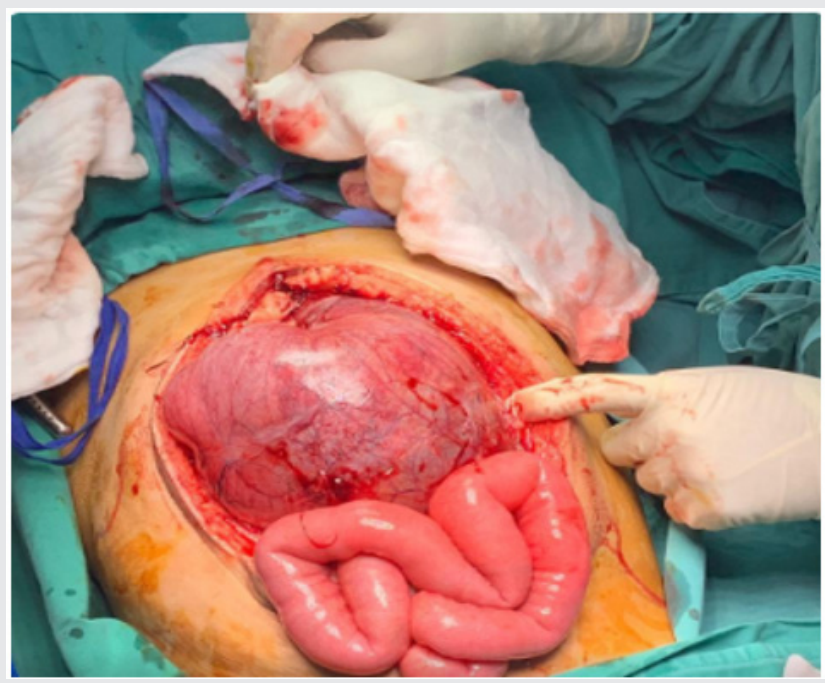

Figure 3: Intra-operative findings.

The focal rounded air-filled cecum may present as a loop with haustral markings resembling a coffee bean; which appears as a dilated bowel loop with an inverted "U" shape converging at the site of torsion, and a thickened central radiopaque line composed by the walls of the two part of the colon that are adjacent to each other. Doppler USG may lead to make a definite diagnosis by showing twisted mesenteric vessels [11], and CT may be more diagnostic by demonstrating cecal distension, cecal apex in left upper quadrant, mesenteric whirl, ileocecal twist, and small bowel distension [12]. An important sign to look for is the "whirl sign" that consists of a whirlpool pattern of swirling structures including collapsed bowel loops, mesenteric fat and engorged ileocecal vessels (Figure 3). This finding, associated to a dislocated enlarged cecum, is acknowledged to be diagnostic of volvulus $[9,13]$. The treatment of caecal volvulus preferred surgical procedure for the treatment of patients with cecal volvulus is right hemicolectomy [7].

\section{Case in Detail}

A 22-year-old man was referred from district clinic for sudden onset abdominal pain, vomiting and constipation and increasing abdominal distension for two days. He is a known case of cerebral palsy and imperforated anus since birth. He had undergone surgery for colostomy, subsequently surgery to correct the imperforated anus and stoma reversal during infancy. However, he is ADL independent. His mother (informant) related a history of having chronic intermittent abdominal pain of lesser intensity along with intermittent constipation for several years. His regular bowel habits consist of constipation for 5 days and loose stools for the next 2-3 days. He usually passes urine and stool in the diapers. He was apparently well till two days prior to the admission when he developed sudden abdominal pain disturbing the nights to sleep. He had been keeping his lower limbs flexed for the pain. He vomited more than 10 times a day after the food which was non-projectile, non- bilious and consisted of food particles. He lost his appetite and had absolute constipation. He was able to pass mixed hard and soft stool after administration of enema in the clinic where he visited one day prior to the admission. On examination, he was alert conscious, co-operative, comfortable under room air and capillary refill time is less than two seconds. His pulse rate was 130 beats per minute, normal volume, regular rhythm, blood pressure $141 / 86 \mathrm{mmHg}$, temperature 38.6 degrees Celsius, respiratory rate 36 breaths per minute but pain score unable to illicit for his poor mental function.

His plasma glucose was $9.1 \mathrm{mmol} / \mathrm{L}, \mathrm{SpO} 298 \%$ under $3 \mathrm{~L}$ 02 nasal prong. Abdominal examination revealed generalised abdominal distension, well healed $10 \mathrm{~cm}$ long horizontal scar at the left iliac region, inverted umbilicus and intact hernia orifices. Palpation revealed generalised abdomen tenderness. There was no rebound tenderness and shifting dullness negative. Digital per rectal revealed no palpable mass and the stool was brownish. Cardiovascular, Respiratory examination were normal. Upon arrival at A\&E, he was kept nil per oral, urinary catheter inserted-100cc straw urine was collected. Initial treatment of fluids, Morphine and antibiotics were administered intravenously. ECG, blood tests for investigation, chest and abdominal X ray was taken. FAST Scan revealed no free fluid. ECG was normal except sinus tachycardia. Full blood count was normal except neutrophilic leucocytosis: TWC $16.3 \times 109 / \mathrm{L}$ (N: 4-10). Renal profile and BUSE were normal except 
mildly high Urea $8.5 \mathrm{mmol} / \mathrm{L}(\mathrm{N}: 3.2-8.2)$ and decreased $\mathrm{Na}+134$ $\mathrm{mmol} / \mathrm{L}$ (136-145). Liver function test was normal except mildly high total Bilirubin 26.8 umol/L (N: <21) Serum Amylase was normal :40 U/L (N:30-118). Chest-X-ray was normal.

Minimal soft stool was obtained on manual evacuation. Clinical diagnosis of Intestinal obstruction secondary to old abdomen surgery scar adhesions was made. Surgical opinion was obtained soon. Patient was then transferred to the surgical ward and urgent CT abdomen was requested. Large amount of impacted stool was removed on another manual evacuation of stool. A Ryle's tube was inserted. No focal lung lesion in the lung bases. Mild left pleural effusion. There is hugely dilated bowel loop seen across the upper abdomen associated with whirlpool sign and small bowel loops seen at the right iliac fossa. Features are suggestive of cecal calculus. There is no bowel wall thickening to suggest bowel ischemia. However, there is mild free fluid seen in the abdomen and pelvis associated with streakiness of the mesenteric fat. The stomach and spleen are displaced by the dilated bowel loop posteroinferiorly. Faecal laden sigmoid colon and rectum noted. The descending colon and part of the transverse colon are collapsed with no bowel related mass seen. No focal lesion in the liver, spleen, adrenals and pancreas. No hepatosplenomegaly. The CBD and intrahepatic ducts are not dilated. There is no calculus in the gall bladder. Both kidneys have normal enhancement. No hydronephrosis. No significant para aortic or pelvic lymphadenopathy. No suspicious bony lesion.

\section{Impression}

Features are suggestive cecal volvulus with mild free fluid. Mild left pleural effusion.

A clinical diagnosis of ceacal volvulus was made and emergency operation was done through midline laparotomy scar.

\section{Intra-operative Findings}

Stomach normal, small bowel not dilated, transverse, descending, sigmoid colon normal 270 degrees, caecal volvulus with grossly dilated caecum, bowel ischemia, adhered to anterior abdominal wall, upon mobilization there is bowel perforation with localised faecal contamination at left hypochondrium- 300cc. Minimal adhesion of sigmoid colon to previous scar- released. Faecal loaded rectum, manual evacuation done. Multiple enlarged mesenteric lymph nodes. Limited right hemicolectomy with double barrel stoma was done. Surgical procedure: Caecum was decompressed via enterotomy. Faecal contamination during mobilisation, peritoneal lavage done. Caecum, ascending colon, ileum mobilised. Limited right hemicolectomy done with double barrel stoma. Drain inserted, abdomen closed in layers. Stoma measured at right iliac fossa. Patient then was transferred to ICU for further management. Culture sensitivity of peritoneal fluid, blood and secretions of endotracheal tube revealed no bacterial growth. He was then transferred to the ordinary ward when he was stable and discharged five days later uneventfully.

\section{Discussion}

In our opinion this patient seems to be suffering from double pathology: Chronic large bowel obstruction due to post-op adhesion, and Cecal Volvulus due to congenital predisposing factors. Radiology plays a role to diagnose caecal volvulus since there is no specific symptoms and signs of caecal volvulus except those of intestinal obstruction. There were classic plain X-ray abdomen of coffee bean sign of caecal volvulus [8-10] and CT abdominal features of caecal volvulus $[12,13]$ in this patient. Mildly low sodium level and high urea changes indicate dehydration due to bowel obstruction. An elevated white blood cell (WBC) count may indicate bowel ischemia, peritoneal infection with or without gut perforation, or systemic sepsis. However, there was no features of sepsis clinically in this patient preoperatively. Therefore, bowel ischemia is the most likely cause of leukocytosis in this case preoperatively. Different treatment and results are reported by Consorti ET and Liu TH [1]: Barium enema sporadic reports of reduction after barium enema. The success rate is unknown. This modality is not usually recommended as a therapeutic option.

Colonoscopy reduction of volvulus by endoscopic approach; the reported success rate is about $30 \%$ and the recurrence rate is unknown. Operative detorsion manual reduction of volvulus by caeliotomy. Mortality 0\%- 25\%; recurrence 0\%-70\%. Caecopexy Fixation of right colon by suturing of caecum and/or ascending colon to lateral parietal peritoneum. Operative mortality $0 \%-30 \%$; recurrence $0 \%-40 \%$. Caecostomy tube placement Fixation of right colon by tube placement into caecum. Operative mortality 0\%-40\%; recurrence 0\%-33\%. Colectomy Resection of involved intestinal segment. Mortality 0\%-39\% with lower mortality in patients treated after 1990. No recurrence has been reported after resection.Given the modest success rate, the potential for colonic perforation, and potential delays in operative treatment associated with unsuccessful reduction, colonoscopy is generally not recommended in the initial treatment of caecal volvulus $[14,15]$. Radiologic diagnoses of cecal volvulus or cecal bascule are also generally considered indications for surgical intervention. The choice of procedure depends on the patient's clinical condition. In severely debilitated patients, cecostomy is a valid option; however, it is associated with a wound infection rate of $40-50 \%$ and a recurrence rate of approximately $2-5 \%$ [7]. Surgery was opted in this case because of generalised abdominal tenderness and leucocytosis indicating bowel ischaemia and right hemicolectomy was carried out. He had uneventful recovery and was discharged after five days of surgery.

\section{Conclusion}

Susceptible persons for caecal volvulus should be advised to seek medical attention and hospital admission in view of need of surgery if they should develop symptoms to suggest intestinal obstruction. 


\section{Conflict}

There is no conflict of interest.

\section{Acknowledgement}

We are thankful to hospital director of Melaka General Hospital for allowing to publish the case. We are also thankful to Prof Krishna Kumar Mallick and Prof Ava Tay and (Dept of Surgery of Melaka Manipal Medical College) for their expert opinion in the evaluation of the case.

\section{References}

1. Consorti ET, Liu TH (2005) Diagnosis and treatment of caecal volvulus. Postgrad Med J 81(962): 772-776.

2. Samiullah Bhatti, Mahmood Ayyaz Khan, Waris Farooka, Butt UI, Rehman UA, et al. (2017) An Unusual Case of Caecal Volvulus due to Appendicitis, Successfully Managed by Caecopexy. Journal of the College of Physicians and Surgeons Pakistan 27(3): 18-20

3. O Mara CS, Wilson TH, Stonesifer GL (1979) Caecal volvulus: Analysis of 50 patients with long-term follow-up. Ann Surg 37(2): 132-136.

4. Ballantyne GH, Brandner MD, Beart RW (1985) Volvulus of the colon: incidence and mortality. Ann Surg 202(1): 83-92.

5. Cole FR (1956) Volvulus of the caecum associated with acute suppurative appendicitis. N Y State J Med 56(13): 2120.

\section{ISSN: 2574-1241}

DOI: 10.26717/BJSTR.2019.19.003268

Aye M. Biomed J Sci \& Tech Res

CC) This work is licensed under Creative

Submission Link: https://biomedres.us/submit-manuscript.php
6. D Egidio A, Lipschitz J (1988) Acute appendicitis associated with caecal volvulus. A report of 2 cases. S Afr J Surg 26(1): 27-28.

7. Thornton SC, Pal N (2018) Drugs \& Diseases, General Surgery.

8. Peterson CM, Anderson JS, Hara AK, Carenza JW, Menias CO, et al. (2009) Volvulus of the gastrointestinal tract: appearances at multimodality imaging. Radiographics 29(5): 1281-1293.

9. Delabrousse E, Sarliève P, Sailley N, Aubry S, Kastler BA, et al. (2007) Cecal volvulus: CT findings and correlation with pathophysiology. Emerg Radiol 14(6): 411-415.

10. Moore CJ, Corl FM, Fishman EK (2001) CT of cecal volvulus: unraveling the image. AJR Am J Roentgenol 177(1): 95-98.

11. Takada K, Hamada Y, Sato M, Fujii Y, Teraguchi M, et al. (2007) Cecal volvulus in children with mental disability. Pediatr Surg Int 23(10): 1011-1014.

12. Rosenblat JM, Rozenblit AM, Wolf EL, Du Brow RA, Den EI, et al. (2010) Findings of cecal volvulus at CT. Radiology 256(1): 169-175.

13. Frank AJ, Goffner LB, Fruauff AA, Losada RA (1993) Cecal volvulus: the CT whirl sign. Abdom Imaging 18(3): 288-289.

14. Madiba TE, Thomson SR (2002) The management of cecal volvulus. Dis Colon Rectum 45(2): 264-267.

15. Friedman JD, Odland MD, Bubrick MP (1989) Experience with colonic volvulus. Dis Colon Rectum 32(5): 409-416.

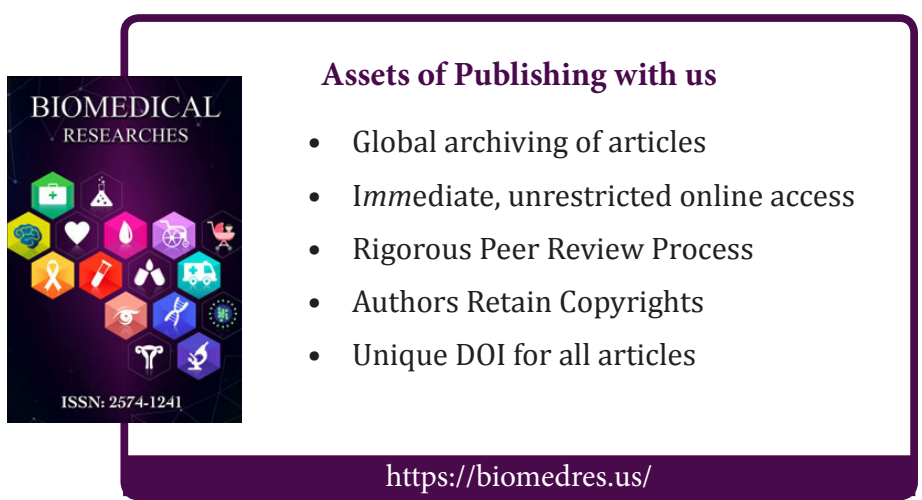

